\title{
The use of Soxhlet extractor for the production of tinctures from plant raw materials
}

\author{
Dmitrii Borodulin ${ }^{1}$, Maksim Prosin ${ }^{1, *}$, Igor Bakin ${ }^{1}$, Boris Lobasenko ${ }^{1}$, Marina Potapova ${ }^{1}$, \\ Aleksei Shalev ${ }^{1}$ \\ ${ }^{1}$ Kemerovo State University, 6, Krasnaya St., 650000, Kemerovo, Russia
}

\begin{abstract}
The most important stage in the production of tinctures and aromatic alcohols is the extraction process. The paper aims to study the process of extracting target compounds from fruit and berry raw materials for the production of alcoholic tinctures. The research was carried out using a Soxhlet extractor. The experimental results were processed using the method of multiple regression analysis. As a result of processing the experimental data, the optimal technological regime parameters for the production of aromatic cranberry alcoholic tincture were selected. The cranberry tinctures produced at these parameters have a high content of target substances and reach maximum scores for taste, aroma and color. The obtained regression equations allow predetermining the quality of the resulting drink with a confidentiality of more than $90 \%$. The use of the Soxhlet extractor allowed reducing the duration of the preparation of the cranberry tincture to 15 minutes, which is by times different from the classic method of tincture production. The use of the Soxhlet extractor allows reducing the consumed amount of solvent and raw materials, due to the almost maximum rate of the extraction. All this, in turn, affects the cost of the final product and the costs of the manufacturer.
\end{abstract}

\section{Introduction}

Alcohol was discovered many centuries ago, and the demand for it has not decreased so far. The market for alcoholic beverages has been filled with various types and varieties, from classic inexpensive varieties to premium drinks.

The French can boast of wine, the Portuguese - of port, the Scots - of whiskey, and the Russians are famous for their variety of tinctures, having long fermented everything that can be fermented. Russia has no equal in the number of varieties of tinctures - alcoholic beverages produced by infusing alcohol of different strength on different ingredients [1].

The main raw materials for producing tinctures have always been plants. Modern manufacturers prefer berries and fruits, but there are also some versions of products made on kernels, fragrant and medicinal herbs, spices, and other components [2-4].

More than 100 types of plant raw materials are used in production of tinctures. The chemical composition of plant raw materials is very complex and diverse: it is not only different species of plants, but also different varieties within the same species that have

\footnotetext{
*Corresponding author: prosinmv@yandex.ru
} 
different chemical composition. The content of certain substances varies in wide ranges, depending on a variety of reasons. First of all, it is affected by the climate, meteorological conditions of the growing season and the quality of the soil. Weather is very important as well, and even the size of the fruits of the same plant affects their chemical composition [4].

Currently, due to the growth of consumer power of the Russian population and the increase in demand for alcoholic beverages, import substitution issues are becoming topical. To reduce the cost of production and expand the product range, it is necessary to use the major raw materials growing in the territory of Siberia [3, 4].

The main stage in the production of tinctures and aromatic alcohols is the extraction process. Today, extraction is one of the major processes in almost all industries, and in the food industry, this process is mostly used in food production $[5,6]$.

As for the mechanization of the extraction process, a large number of extractors for the solid-liquid system of periodic and continuous action have been designed so far. All of them differ in efficiency, energy consumption and applicability in a particular production process [5-7].

Increasing the speed of technological processes and productivity of technological equipment while improving the quality of the final product are the main goals set by designers and developers when creating new constructions of devices and machines or upgrading the existing ones. Therefore, the research and development of new equipment for efficient processes is an highly relevant task for technical sciences [7, 8].

This work aims to study the process of extracting target components from fruit and berry raw materials for the production of alcoholic tinctures, and to select the rational technological regime parameters of the process.

\section{Materials and methods}

Cranberries, which are widely spread over Siberia and have many useful properties, were chosen as the object of research. Cranberries are often used for making various tinctures. This berry easily withstands frost: therefore, ripening in autumn, it is well preserved until spring. Cranberries contain a huge amount of citric and quinine acid, the berries are rich in vitamins $\mathrm{C}, \mathrm{B} 1, \mathrm{~B} 2, \mathrm{~B} 5, \mathrm{~B} 6, \mathrm{PP}$ and the rarer vitamin $\mathrm{K} 1$. Cranberries are used for medicinal purposes as an antipyretic, diuretic, and bactericidal agent $[9,10]$.

It is desirable that the berries used for making tinctures should be ripe and not spoiled. The chemical composition of cranberries may vary depending on a number of factors: the area of growing, climate and picking time [10].

Cranberries can be stored for a long time, but the correct storage conditions must be observed.

A water-alcohol solution based on premium-class ("Luxe") ethyl alcohol of "Luxe", meeting the standards of GOST 5962-2013 was used as the extractant. The premium-class ("Luxe") ethyl alcohol is a grain ethyl alcohol of the highest quality obtained by rectification from wheat, barley, rye grain or a mixture of grains. Most of the vodka and liquors produced in Russia are made from premium-class ("Luxe") ethyl alcohol. It is a transparent colorless liquid, without foreign odors and tastes. The content of ethyl alcohol is $96.5 \%$. The water used in research meets the requirements of GOST 6709-72.

We believe the Soxhlet extractor to be the most suitable apparatus for producing tinctures. The main advantage of this apparatus is that pure alcohol is fed inside the extractor; the alcohol is not saturated with the extracted substance, which contributes to a more effective enrichment with aromatic oils and esters. The Soxhlet extractor is designed for obtaining extracts from various raw materials and fast flavoring of strong drinks [11].

The classic method of producing tinctures is a time-consuming process, while the Soxhlet extractor allows reducing the time of preparation of tinctures from several weeks, 
or even months, to several hours. The principle of its operation is based on repeated processing of aromatic raw materials with a small volume of the extracting solvent (a strong alcohol-containing solution), as a result of which the aromatic compounds are dissolved from the raw materials, forming an extract.

The advantages of the Soxhlet extractor are: simple and clear design, production process continuity, ease of visual monitoring of the process, a low flow of solvent and the possibility of its reuse after stripping and distillation.

Figure 1 shows the design of the Soxhlet extractor. It consists of a distillation still (1) with a siphon arm (5) installed in it, which has a distillation arm (3) and a siphon (4). The thimble (6), into which the extract raw materials are loaded, is installed in the siphon. The extractor chamber (2) is closed with the thimble cover (7). The the reflux condenser (8) is fixed to the extractor chamber with of a connection valve. The cold water for cooling the studied liquid flows in through the inlet nozzle (9), and the used water flows out through the outlet nozzle (10).

When the liquid in the distillation still (1) boils, the alcohol-containing vapour rises through the distillation arm (3), the vapour reaches the reflux condenser (8), it is cooled and turns into the liquid, which drips into the thimble (6) and wets the raw materials loaded in it. As the thimble is filled with the solvent, the aromatic compounds are extracted into the alcohol. After the thimble is completely filled with alcohol, the liquid reaches the upper level of the siphon (4), the thimble chamber is emptied. The solution flows down the siphon arm (5) into the distillation still (1). Depending on the set experimental conditions, this cycle is repeated again. Thus, the extraction process may be repeated many times [11].

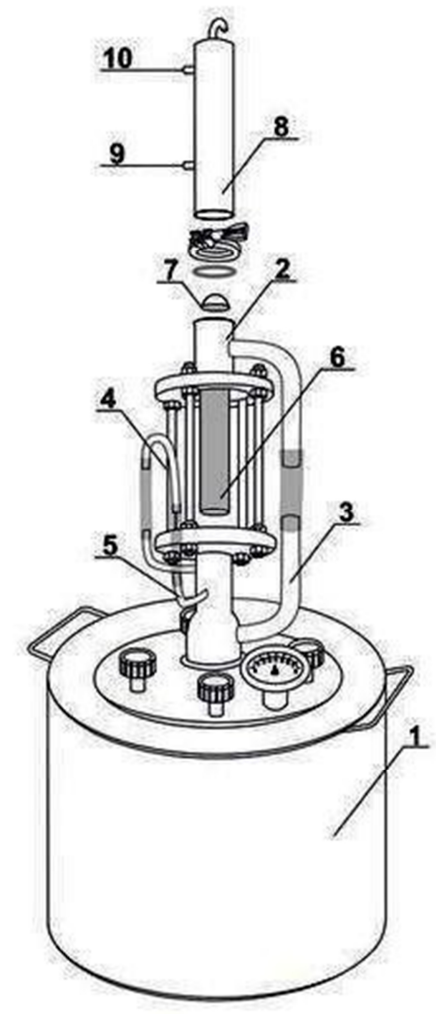

Fig. 1. Soxlhet extractor: 1 - distillation still, 2 - extractor chamber, 3 - distillation arm, 4 - siphon, 5 - siphon arm, 6 - thimble, 7 - thimble cover, 8 - reflux condenser, 9 - cooler inlet nozzle, 10 - cooler outlet nozzle. 
The generally accepted methods for evaluating the quality of samples of alcoholic beverages were used within the research [12].

The main indicator for evaluating the effectiveness of the apparatus used is the solid content. The solid content in the experimental samples of tinctures was determined by drying using the laboratory oven (model 35/250-500-P-Standard) [13].

It is not enough to evaluate only the physical and chemical parameters of the resulting tinctures, which is also true for evaluating other products of the food industry. Thus, tasting evaluation was performed to determine the parameters of taste, aroma and color. The quality of alcoholic beverages was assessed using well-known standard methods based on the results of the tasting panel score [13, 14].

The results were mathematically processed using the method of multiple regression analysis to determine the effects of variable parameters on the quality of the resulting drink $[15,16]$.

\section{Results and discussion}

To determine the rational modes of operation of the Soxhlet extractor, experimental studies were conducted to assess the effects of the strength of the water-alcohol solution $\mathrm{K}$, the volume of loading berries into the thimble VST and the duration of extraction $\tau$, on the solid content $\mathrm{C}$ in the resulting tincture.

The following parameters were varied during the experiments: the strength of solution $(\mathrm{K})$ varied in the range of $40-50^{\circ} \mathrm{C}$; the volume of berries loaded into the thimble $\mathrm{V}_{\text {th }}$ varied in the range of $62-186 \mathrm{~cm}^{3}$; the duration of extraction process $\tau$ varied in the range of $10-20$ minutes. The values of the parameters by level are shown in Table 1.

Table 1. The values of the parameters by level.

\begin{tabular}{|c|c|c|c|}
\hline Level & $\mathbf{V}_{\text {th }}, \mathbf{c m}^{3}$ & $\boldsymbol{\tau}, \mathbf{m i n}$. & Strength of solution (K) \\
\hline Upper level & 186 & 20 & 50 \\
\hline Lower level & 62 & 10 & 40 \\
\hline Center point & 124 & 15 & 45 \\
\hline Interval & 62 & 5 & 5 \\
\hline
\end{tabular}

The experimental data for the studied tinctures were processed in the integrated system of complex statistical analysis "Statistica 10". As the result, the regression surfaces for the solid content and the equations describing them were obtained. Figure 2 shows the response surfaces for the regression analysis of the solid content.

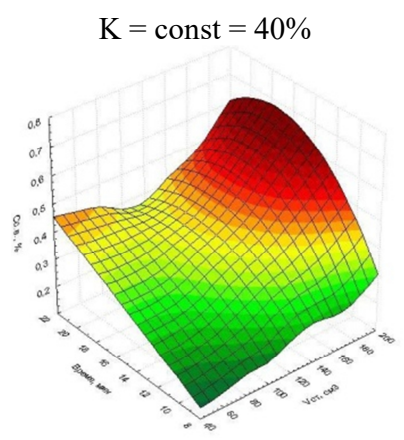

A

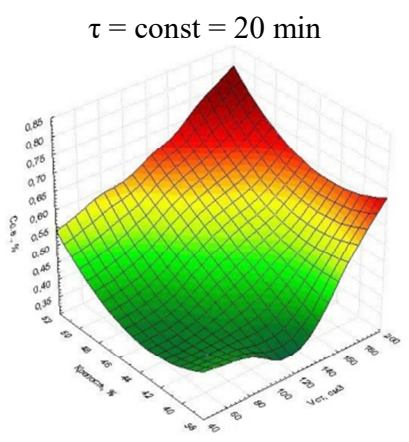

B

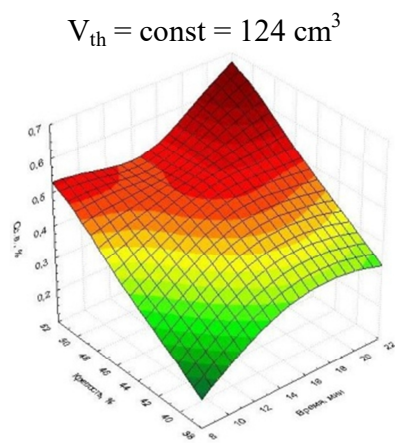

C

Fig. 2. Response surfaces for the solid content.

The regression equations describing the response surfaces are: 
A) $\mathrm{S}_{40}=-0.3333-0.013 \mathrm{~V}_{\text {th }}+0.0767 \tau-0.0082 \tau^{2}$

B) $\mathrm{S}_{20}=2.5111-0.0005 \mathrm{~V}_{\text {th }}-0.1067 \mathrm{~K}+0.0013 \mathrm{~K}^{2}$

C) $\mathrm{S}_{124}=-1.9222+0.3 \tau+0.0767 \mathrm{~K}-0.0007 \tau^{2}-0.00007 \mathrm{~K}^{2}$

To determine the effect of all 3 studied parameters on the solid content, the general equation for the performed experiments was obtained, taking into account all three factors:

$$
S=0.9472+0.00164 V_{t h}+0.0543 \tau-0.6666 K-0.00129 \tau^{2}+0.0009 K^{2}
$$

From equation (1), we can conclude that the solid content is more affected by the time of the extraction process. The experiment duration of 20 minutes allows the cycle to be repeated 4 times, thereby more valuable compounds from cranberries can be.

An important element of regression analysis is the assessment of the adequacy of the model, which is based on the analysis of deleted residues. The residual plot (Fig. 3) shows that their values are normally distributed, since all points deviate insignificantly from the straight line, and most of them do not go beyond the boundary conditions. Therefore, the resulting model describes the experimental data adequately, with more than $95 \%$ confidentiality.

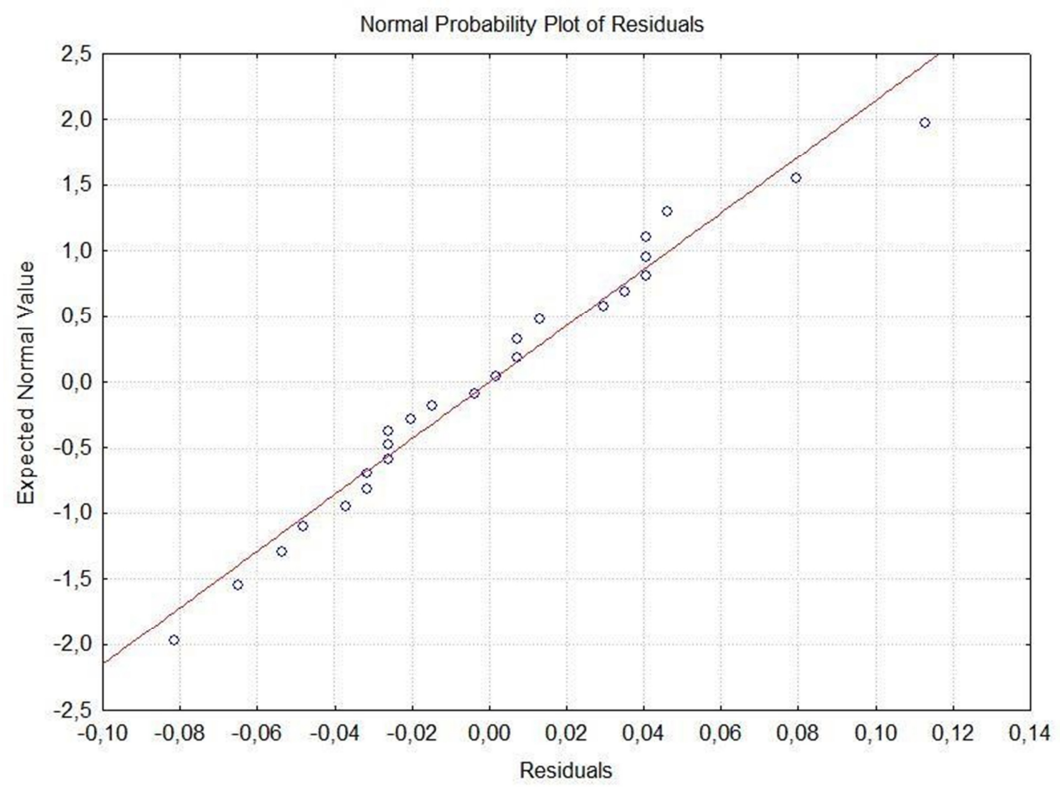

Fig. 3. Residual plot for the solid content in tinctures.

Evaluating the solid content, we can conclude that the best parameters will be obtained at the solution strength ranging within $48-50 \%$, the volume of berries loaded ranging within $180-200 \mathrm{~cm}^{3}$, and in the process duration ranging within 16-20 minutes. These parameters ensure a high solid content. Control experiments conducted at these parameters confirmed the calculated values.

The next step in determining the quality of the resulting tinctures was a tasting evaluation of their taste, aroma and color. To do this, a panel of five people scored the experimental samples. The experimental data were processed in the integrated system of complex statistical analysis "Statistica 10 " as well. As a result, the regression surfaces presented in Figures 4, 6 and 8 were obtained. 


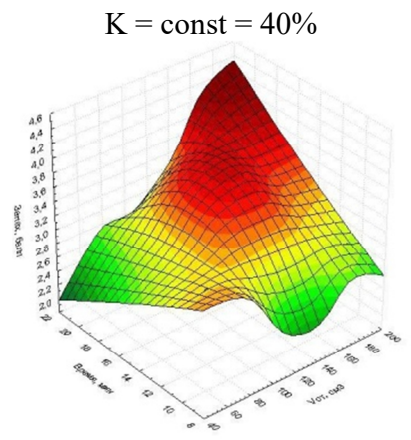

A

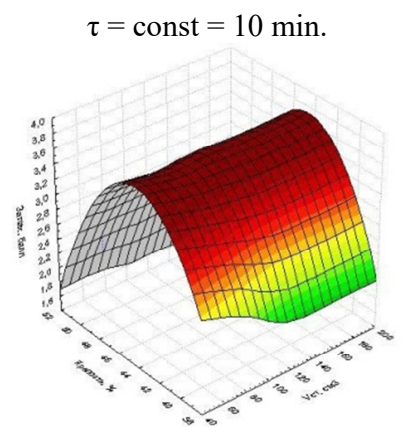

B

$$
\mathrm{V}_{\mathrm{th}}=\mathrm{const}=124 \mathrm{~cm}^{3}
$$

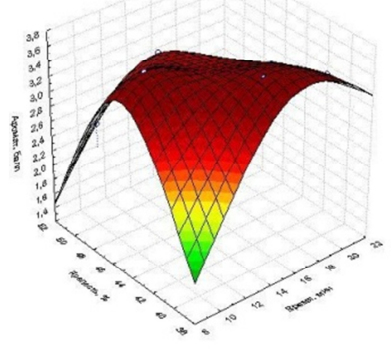

$\mathrm{C}$

Fig. 4. Response surfaces for the aroma of tinctures.

The regression equations describing the response surfaces are:
A) $\mathrm{A}_{40}=2.7944-0.0055 \mathrm{~V}_{\mathrm{th}}+0.0633 \tau+0.0014 * \mathrm{~V}_{\mathrm{th}} * \tau-0.0067 \tau^{2}$
B) $\mathrm{A}_{10}=-65.7833-0.0149 \mathrm{~V}_{\text {th }}+3.17 \mathrm{~K}+0.0002 * \mathrm{~V}_{\text {th }} * \mathrm{~K}-0.036 \mathrm{~K}^{2}$
C) $\mathrm{A}_{124}=-27.7194+0.6817 \tau+1.2217 \mathrm{~K}-0.0153 \tau^{2}-0.005^{*} \tau^{*} \mathrm{~K}-0.0133 \mathrm{~K}^{2}$

To determine the effect of all 3 studied parameters on the aroma of tinctures, the general equation for the performed experiments was obtained, taking into account all three factors:

$$
A=-346646-0.0088 V_{t h}-0.096 \tau+1.8035 K+0.00077^{*} V_{t h}{ }^{*} \tau-0.02056 K^{2}
$$

From equation (2), we can conclude that the flavor of cranberry tinctures is more affected by the strength of the water-alcohol solution, and to a lesser extent by the volume of berries loaded.

The residual plot for the solid content in tinctures (Fig.5) shows the degree of confidentiality of the description of the experimental data of more than $95 \%$.

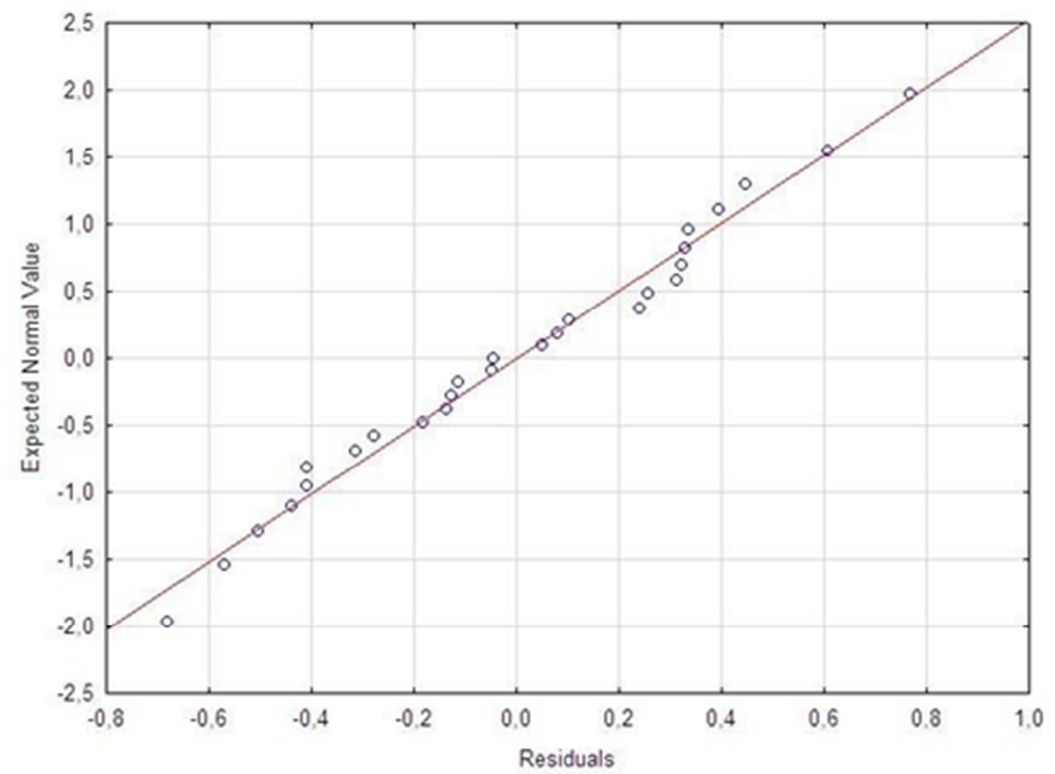

Fig. 5. Residual plot for the aroma of tinctures. 
Evaluating the aroma of cranberry tinctures, we can conclude that the best indicators will be reached at solution strength ranging within $42-46 \%$, the volume of berries loaded ranging within $130-185 \mathrm{~cm}^{3}$, and the process duration ranging within 15-19 minutes. These parameters ensure a rich aroma.

The response surfaces for the taste of tinctures are shown in Fig. 6.

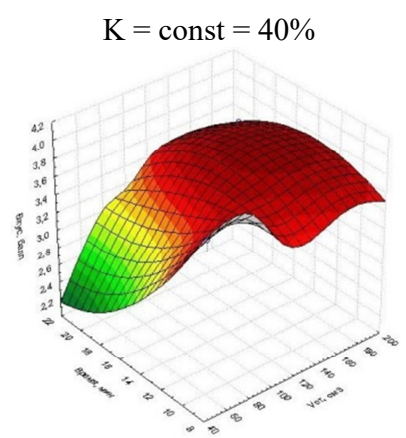

A

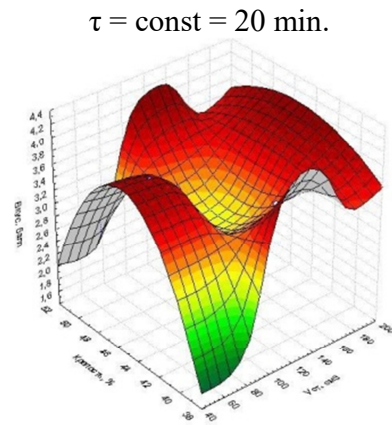

$\mathrm{B}$

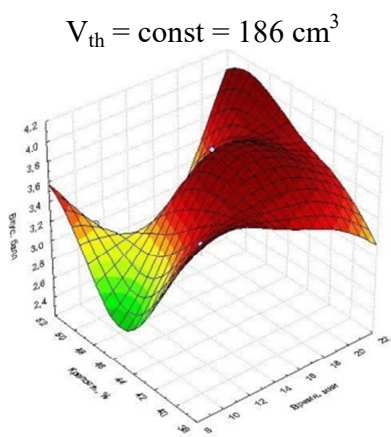

$\mathrm{C}$

Fig. 6. Response surfaces for the taste of tinctures.

The regression equations describing the response surfaces are:
A) $\mathrm{T}_{40}=3.5833+0.0098 \mathrm{~V}_{\text {th }}-0.0667 \tau+0.0007 * \mathrm{~V}_{\text {th }} * \tau-0.002 \tau^{2}$
B) $\mathrm{T}_{20}=-13.4833-0.0015 \mathrm{~V}_{\text {th }}+0.7367 \mathrm{~K}-0.008 \mathrm{~K}^{2}$
C) $\mathrm{T}_{186}=-0.3722-0.2067 \tau+0.28 \mathrm{~K}-0.0007 \tau^{2}+0.006 * \tau * \mathrm{~K}-0.0047 \mathrm{~K}^{2}$

To determine the effect of all 3 studied parameters on the taste of tinctures, the general equation for the performed experiments was obtained, taking into account all three factors:

$$
T=21.8708+0.0036 V_{t h}-0.8353 K+0.00928 K^{2}
$$

The equation (3) shows that the taste of cranberry tinctures is more affected by the strength of the water-alcohol solution, and to a lesser extent by the volume of berries loaded in the Soxhlet extractor $\left(\mathrm{V}_{\mathrm{th}}\right)$, while the duration of the extraction process has no effect on the taste of the drink.

The residual plot for the solid content in tinctures (Fig.7) shows the degree of confidentiality of the description of the experimental data of more than $90 \%$. 


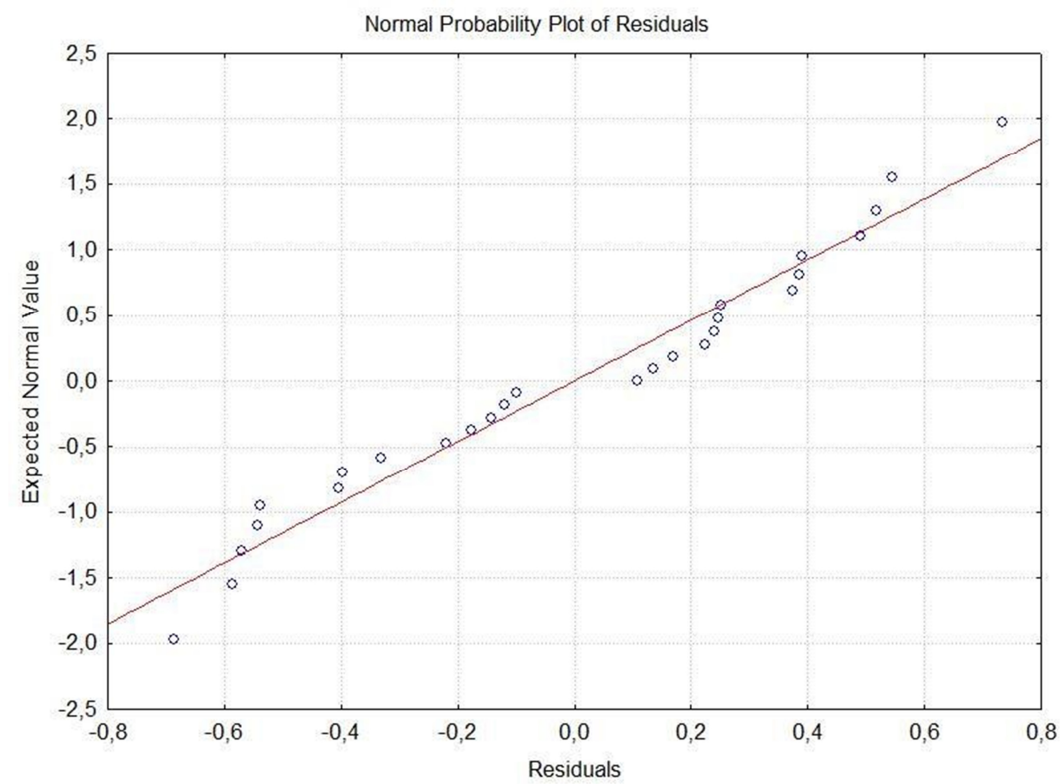

Fig. 7. Residual plot for the taste of tinctures.

Evaluating the taste of cranberry tinctures, we can conclude that the best indicators will be reached at solution strength ranging within $44-50 \%$, the volume of berries loaded ranging within $145-185 \mathrm{~cm}^{3}$, and the process duration ranging within $15-20$ minutes. These parameters ensure a rich taste of the tincture. Control experiments conducted at these parameters confirmed the calculated values of the taste of tinctures.

The response surfaces for the colour of tinctures are shown in Fig. 8.

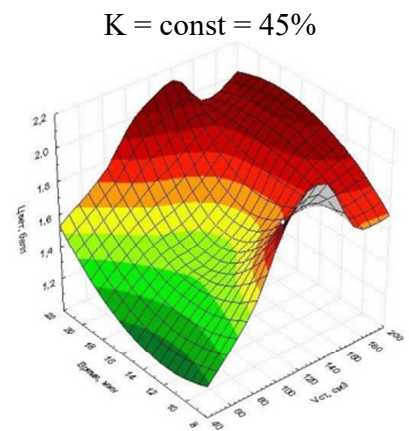

A

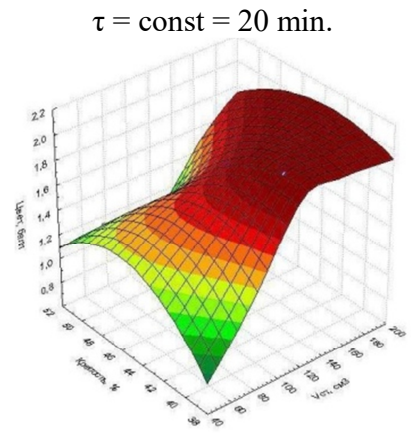

$\mathrm{B}$

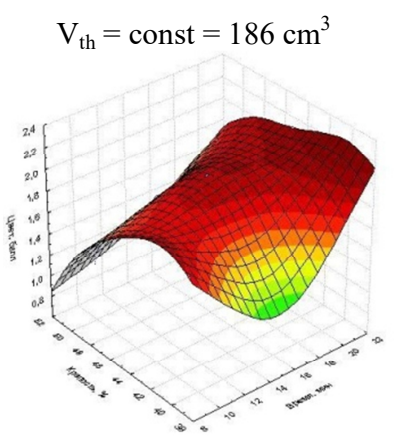

$\mathrm{C}$

Fig. 8. Response surfaces for the colour of tinctures.

The regression equations describing the response surfaces are:
A) $\mathrm{C}_{45}=1.5667+0.0078 \mathrm{~V}_{\text {th }}-0.1067 \tau+0.004 \tau^{2}$
B) $\mathrm{C}_{20}=-10.6667+0.0151 \mathrm{~V}_{\text {th }}+0.53 \mathrm{~K}-0.0002 * \mathrm{~V}_{\text {th }} * \mathrm{~K}-0.006 \mathrm{~K}^{2}$
C) $\mathrm{C}_{186}=-17.4194-0.245 \tau+0.9517 \mathrm{~K}+0.0074 \tau^{2}+0.003 * \tau * \mathrm{~K}-0.0113 \mathrm{~K}^{2}$

To determine the effect of all 3 studied parameters on the colour of tinctures, the general equation for the performed experiments was obtained, taking into account all three factors:

$$
C=-10.5616+0.0117 V_{t h}-0.0739 \tau+0.5545 K-0.00003 * V_{t h}{ }^{2}+0.00298 \tau^{2}-0.0064 K^{2}
$$


From equation (4), we can conclude that the colour of cranberry tinctures will be more influenced by the strength of the water-alcohol solution, and to a lesser extent by the volume of berries loaded.

The residual plot for the solid content in tinctures (Fig.9) shows the degree of confidentiality of the description of the experimental data of more than $95 \%$.

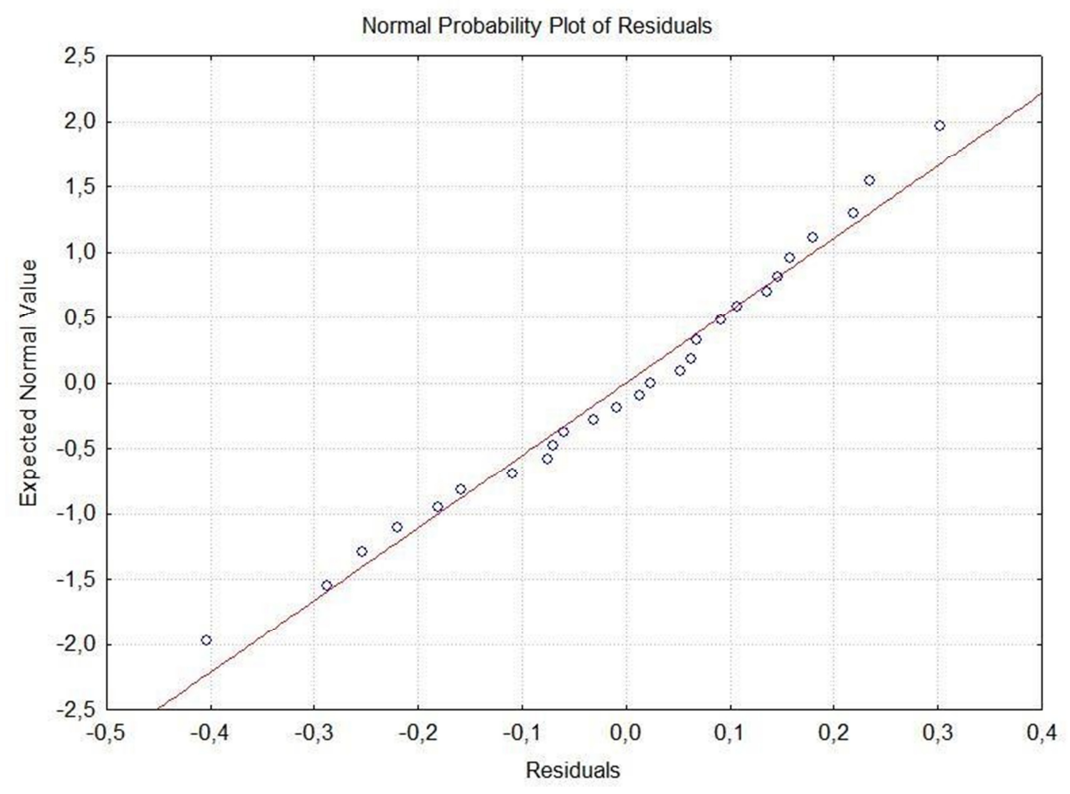

Fig. 9. Residual plot for the colour of tinctures.

Evaluating the colour of cranberry tinctures, we can conclude that the best indicators will be reached at solution strength ranging within $42-46 \%$, the volume of berries loaded ranging within $160-185 \mathrm{~cm}^{3}$, and the process duration ranging within 14-18 minutes.

\section{Conclusions}

The performed experiments allow us to evaluate the efficiency of cranberry tinctures production using the Soxhlet extractor.

As a result of processing of the experimental data, the following optimal technological regime parameters of production were selected: namely $\mathrm{V}_{\mathrm{th}}=165 \mathrm{~cm}^{3}, \mathrm{~K}=45 \%, \tau=15 \mathrm{~min}$. The cranberry tinctures produced at these parameters have a high content of target substances and reach maximum scores for taste, aroma and color [14].

The obtained regression equations allow predetermining the quality of the resulting drink with a confidentiality of more than $90 \%$.

The use of the Soxhlet extractor allowed reducing the duration of the preparation of the alcoholic cranberry tincture to 15 minutes, which is by times different from the classic method of tincture production. The use of the Soxhlet extractor allows reducing the consumed amount of solvent and raw materials, due to the almost maximum rate of the extraction of the target compounds from fruit and berry raw materials. All this, in turn, affects the cost of the final product and the costs of the manufacturer, as well as their economic performance. 


\section{References}

1. I. Tonutti, Flavour and fragrance journal 25(5), 341-350 (2010) DOI: https://doi.org/10.1002/ffj.2001

2. J.Y. Zhang, Antioxidants 8(5) (2019) DOI: https://doi.org/10.3390/antiox8050144

3. M. Carlsen, Nutrition Journal 9(1), 15-23 (2010) DOI: https://doi.org/10.1186/14752891-9-3

4. Yu. Golubtsova, Foods and raw materials 4(2), 4-15 (2016) DOI: http://doi.org/10.21179/2308-4057-2016-2-4-15

5. E. Safonova, Advances in Engineering Research 151, 610-614 (2018) DOI: https://doi.org/10.2991/agrosmart-18.2018.114

6. R. Subramanian, Arabian journal of chemistry 9, 537-540 (2016) DOI: https://doi.org/10.1016/j.arabjc.2011.06.022

7. D. Borodulin, Advances in Engineering Research 151, 578-583 (2018) DOI: https://doi.org/10.2991/agrosmart-18.2018.108

8. D. Borodulin, Storage and Processing of Farm Products 4, 141-453 (2019) DOI: https://doi.org/10.36107/spfp.2019.154

9. A. Karlsons, Agronomy research 16(2), 454-463 (2018) DOI: https://doi.org/10.15159/AR.18.039

10. K. Hurkova, Food chemistry 284, 162-170 (2019) DOI: https://doi.org/10.1016/j.foodchem.2019.01.014

11. A. Konga, Biofuels 8(1), 29-35 (2017) DOI: https://doi.org/10.1080/17597269.2016.1196328

12. E. Egorova, Food and Raw Materials 5(1), 144-154 (2017) DOI: http://doi.org/10.21179/2308-4057-2017-1-144-153

13. L. Dyshlyuk, Journal of Food Process Engineering 40(1), 54-67 (2017) DOI: https://doi.org/10.1111/jfpe.12331

14. R. Evangelista, Industrial Crops and Products 29(1), 189-196 (2009) DOI: https://doi.org/10.1016/j.indcrop.2008.04.024

15. G. Rudoy, Pattern recognition and image analysis 26(23), 608-616 (2016) DOI: https://doi.org/10.1134/S1054661816030172

16. J. Wu, Journal of Electronics and Information Technology 40(5), 1195-1201 (2018) DOI: https://doi.org/10.11999/JEIT170624 